\title{
Seesaw terrestrial wetting and drying between eastern and western Australia
}

\author{
Ajiao Chen ${ }^{1}$, Huade Guan ${ }^{1 *}$, Okke Batelaan ${ }^{1}$ \\ 1 National Centre for Groundwater Research and Training, College of Science and Engineering, Flinders \\ University, Adelaide, SA 5001, Australia \\ *Huade Guan: huade.guan@flinders.edu.au.
}

\begin{abstract}
Australia, the driest inhabited continent, is prone to natural disasters, such as droughts, floods, bushfires and heatwaves. Strong climate variability causes regular threats to water supply, agriculture and the environment. Improving our insight into changes in hydroclimatic patterns is required to provide useful information for society. Previous studies mainly focused on the causes of extreme wet or dry events in specific periods and their impacts on agriculture and ecosystems. An understanding of long-term spatio-temporal patterns of wetting and drying in Australia is still lacking. Here we show, based on analyses of GRACE satellite derived terrestrial water storage (TWS) and extended datasets, that there are four continuous periods of seesaw wetting and drying between eastern and western Australia in the past five decades. The seesaw phenomenon is characterized by eastern Australia gaining water, while western Australia is losing water, and vice versa. Strong La Niña induced continent-wide wetting, resets this pattern, leaving each seesaw to last for $11 \pm 5$ years. We provide one possible mechanism related to vegetation response to climate variability and its feedback on hydrological processes to explain the seesaw resetting pattern. The identified reoccurring seesaw pattern indicates that society would need to become more adaptive in managing forest, water, and disaster risks in the wake of a next strong La Niña induced continent-wide wetting in Australia.
\end{abstract}

\section{Significance Statement}

Large-scale wetting/drying in Australia is commonly thought to be controlled by precipitation. Here we report for the first time a vegetation mediated seesaw wetting/drying phenomenon between eastern and western Australia. The seesaw phenomenon is characterized by eastern Australia gaining water, while western Australia is losing water, and vice versa. It is reset by strong La Niña induced continent-wide wetting. The seesaw phase seems dependent on vegetation cover anomaly prior to the strong La Niña event, and can be explained by subsequent vegetation and soil moisture interactions. This finding provides society with valuable reference for managing forest, water, and disaster risks in the wake of a next strong La Niña induced continent-wide wetting in Australia.

\section{Introduction}

Australia is the driest inhabited continent on Earth and among the areas of the most variable rainfall in the world $(1,2)$. Its hydroclimatic variations play an important role in the global carbon and water cycles $(3,4)$, occurrence of natural hazards $(5,6)$, and agricultural productivity (7). Droughts occurring in Australia during 2000-2009 were reported to reduce global terrestrial net primary production (8). Poulter et al. (9) indicated that the global land carbon sink anomaly triggered by the 2010-11 La Niña event was largely due to an enhanced ecosystem productivity across the Southern Hemisphere, particularly in Australia. Frequent droughts cause agricultural losses $(10,11)$, contribute to bushfires $(12)$ and exacerbate heatwaves $(13,14)$, and consequently impact the economy and society significantly (11). This continent is also prone to floods, which lead to serious 
casualties and economic losses (5). Hence, furthering our understanding and monitoring of wet and dry conditions in Australia is urgently needed for water, agriculture, and disaster risk management.

Previous studies mainly focused on the causes of droughts or floods in discrete periods and their impacts on ecosystems and society $(5,6,11,15,16)$. Xie et al. (4) recently investigated the wet and dry evolution across continental Australia based on Gravity Recovery and Climate Experiment (GRACE) terrestrial water storage (TWS) dataset (2002-2014). They revealed three geographic zones with distinct TWS responses and attributed them to different large-scale teleconnections. If such regional differentiated TWS responses hold over a long period, it would provide society useful information for climate-adaptive water resource planning and management. In this study, we aim to reveal long-term spatio-temporal patterns of TWS in Australia, and to examine how these patterns are associated with climate variability and land surface processes.

\section{Results and discussion}

\section{Main spatial patterns of TWS variation in Australia}

The space-time fields of monthly Australia TWS anomaly from three datasets (14-year original JPL GRACE TWS $(17,18)$ and reconstructed GRACE TWS of 31 (19) and 114 years (20) have been decomposed by empirical orthogonal functions (EOFs). We focus on the first two modes since they explain more than $50 \%$ of the total variance. The three datasets exhibit similar spatial patterns (Fig. 1). Almost all grid cells (more than 90\%) show consistent phase in terms of spatial pattern EOF1 (Fig. 1 (a) (c) (e)) indicating that the wet and dry alternation has consistency over the whole continent, although the north and east parts have higher variability. This EOF mode explains more than $30 \%$ of the total TWS variability in Australia. The spatial pattern EOF2 clearly delineates two zones, eastern and western Australia, with opposite behavior (Fig. 1 (b) (d) (f)). This mode is defined as an east-west opposite pattern, which explains around $20 \%$ of the total variation of the TWS anomaly in Australia. The dashed line in Fig. 1 is drawn based on the average results from the three datasets of different year ranges. This line is coincident with the boundary of the western plateaus and central plains of the Australian continent.

\section{Four consecutive seesaw wetting and drying phases between eastern and western Australia in the past five decades}

The temporal signal of the first decomposed mode (PC1) reflects the wet (positive PC1) and dry (negative $P C 1)$ conditions in terms of average TWS anomaly over the whole continent. The continent-wide wetting and drying are closely related to large-scale ocean-atmosphere dynamics. As shown in Fig. 2, continent-wide wetting episodes represented by positive phases of $P C 1$ generally correspond to positive phases of $S O I$ ( $L a$ Niña episodes). We use pairs of dashed lines to mark the La Niña induced continent-wide wetting episodes, which are bounded by the time points when average TWS anomaly in Australia starts to increase, triggered by strong La Niña, and when it returns to equilibrium. Nine La Niña induced continent-wide wetting episodes are identified during 1901-2014 (W1: Dec 1903-Jan 1905, W2: Mar 1910-Feb 1911, W3: Nov 1916-Nov 1918, W4: Dec 1949-Nov 1951, W5: Feb 1956-Dec 1957, W6: Jul 1973-Mar 1980, W7: Apr 1989-Sep 1989, W8: Aug 1998-Jun 2002, W9: Apr 2010- Jan 2013, see Fig. 2 (a)). As this 114-year reconstructed GRACE TWS dataset is the mean of 100 ensemble members, uncertainty in $P C 1$ is quantified by \pm 1 standard deviation of 100 PC1 time series derived from the ensemble members (Fig. 2 (a)).

As the eastern and western parts of Australia have opposite phases in EOF2 (Fig. 1 (b) (d) (f)), they are supposed to behave oppositely in terms of TWS variation. The periods in-between the La Niña induced 
continent-wide wetting episodes, show piece-wise linear trends of the regional average TWS anomaly in the eastern and western parts of Australia (Fig. 3 (a)). Four consecutive opposite TWS trends between eastern and western Australia are observed during the past five decades (1958-2010). In those four consecutive interval periods, changes of regional average TWS anomaly between the two parts are significantly different at 0.05 significance level (tested by analysis of covariance). This phenomenon is identified as seesaw wetting and drying between these two geographical parts of Australia. The seesaw is characterized by eastern Australia gaining (losing) water, while the west is losing (gaining) water. It appears that a seesaw is reset by a continent-wide wetting episode, turning an earlier wetting (drying) side to lose (gain) water. Another seesaw phenomenon is observed during the 1910s. These five seesaw periods last for $11 \pm 5$ years. Uncertainty in regional average TWS time series is represented by the shaded area in Fig. 3 (a). Uncertainty of the consecutive opposite trends in the last four interval periods is quantified in Fig. 3 (b), the average linear trends of the east and west part derived from 100 ensemble members are significantly different at 0.05 significance level.

Input data of both models for reconstructing the 31- and 114-year TWS include precipitation and temperature information. Data of those two climate variables are of low quality for most of the 114-year time series.

Therefore, we repeated above analysis with the 31-year reconstructed TWS data (1985-2015) for comparison. Three La Niña induced continent-wide wetting episodes (Apr 1989-Jan 1990, Aug 1998-Jun 2002, and Aug 2010- Feb 2013) are marked in Fig. 2 (b), which respectively correspond to W7-9 shown in Fig. 2 (a), although minor differences exist. The regional average TWS anomaly of the eastern and western parts of Australia from 1985 to 2015 are plotted in Fig. 3 (c). Two pairs of opposite trends (all are significant at 0.05 significance level) are observed in the interval periods which correspond to the last two seesaws shown in Fig. 3 (a) respectively.

No seesaw phenomenon is observed if the interval between two La Niña induced continent-wide wetting episodes is too short (1905-1910) or too long (1918-1949). A positive Interdecadal Pacific Oscillation (IPO) phase dominated during 1922-1944 (21), which may have suppressed the occurrence of a La Niña event and/or moderate the La Niña effect (22-26). Indeed, from 1918 to 1949, only one moderate La Niña occurred (1938-1939), but it did not induce continent-wide wetting (Fig. 2 (a)). The length of a continent-wide wetting episode (reflected in the PC1 response, Fig. 2) appears to be associated with the intensity and duration of the La Niña events, as well as the conjunction effects from other oscillations. The wetting episode W7 is short (10 months), which is likely due to the modulation effect of the positive IPO phase (1978-1998) (21). Different from the situation in the previous positive IPO (1922-1944), the La Niña in W7 is strong enough to lead to a continent-wide wetting and reset the seesaw between eastern and western Australia. Here, the La Niña coincident with a negative Indian Ocean Dipole (IOD) (Fig. S1) may explain this situation. Negative IOD usually enhances a La Niña effect, it can also lead to a wetter than normal condition in western and southern Australia $(27,28)$.

\section{Independent evidence for TWS seesaw between eastern and western Australia}

A soil moisture dataset for 1901-2014 provided by Australian Water Availability Project (AWAP) $(29,30)$ also shows a seesaw pattern (Fig. S2 (a)) consistent with that of TWS shown in Fig. 3 (a). The agreement between the results based on two independent data sources supports the validity of the TWS seesaw between the two parts of Australia, because changes in soil moisture are typically the largest component of terrestrial water storage variation (31). 
In addition, as ecosystems are mostly water limited in Australia, changes of terrestrial water storage should have led to and be reflected in vegetation responses. Global Inventory Monitoring and Modeling System (GIMMS) Normalized Difference Vegetation Index (NDVI) data is available from July 1981 to 2015, which provides an opportunity to verify the most recent two TWS seesaw phenomena (Feb 1990-Jul 1998 and Jul 2002- Jul 2010, following the intervals identified based on 31-year reconstructed TWS data). Linear NDVI trends during those two seesaws at each grid cell are shown in Fig. 4 (a) and (b) respectively. A marked eastwest difference in NDVI trends can be observed for both periods. During Feb 1990-Jul 1998, NDVI anomaly increases in the western region and decreases in the eastern region of Australia (Fig. 4 (a)), which is consistent with TWS wetting in the west and drying in the east (Fig. 3 (c)). During Jul 2002- Jul 2010, NDVI anomaly (Fig. 4 (b)) also shows consistent trends with TWS (Fig. 3 (c)). Similar to Fig. 3 (c), we plot the regional average NDVI anomaly of eastern and western Australia from 1985 to 2015 in Fig. 4 (c). During the interval between W7 and W8, the regional average NDVI anomaly significantly increases in the west and slightly decreases in the east. During the interval between W8 and W9, average NDVI anomaly significantly increases in the east and slightly decreases in the west. Changes of regional average NDVI anomaly between the two parts are significantly different in both interval periods (Fig. 4 (c)). These NDVI seesaws, consistent with those of TWS ones, between the western and eastern parts of Australia, provide strong support for the seesaw pattern of TWS variation in Australia.

\section{Possible mechanism of the Australia TWS seesaw phenomenon}

For the four consecutive TWS seesaws between eastern and western Australia, the seesaw state seems to be reset by a continent-wide wetting episode. In other words, if a region was gaining water before the wetting episode, it would be losing water after that. Since terrestrial water storage is mainly recharged by precipitation, we attempted to explain the seesaw phenomenon of TWS by looking into precipitation patterns. The regional average precipitation (provided by AWAP) in the eastern and western parts of Australia during 1901-2014 is plotted in Fig. S2 (b). Opposite trends between two parts are shown for the last three intervals, however, most of them do not pass the significance test at 0.05 significance level. Thus, large-scale precipitation variability, likely associated with climate teleconnections, cannot explain the four consecutive TWS seesaws.

Here we provide one possible mechanism related to dynamic woody vegetation and soil moisture interactions, which appears to explain the seesaw resetting pattern (Fig. 5). For a region with an increasing TWS (the lower blue arrow in Fig. 5) during a seesaw period, vegetation cover tends to be well developed prior to a wetting episode. Such an improved vegetation cover makes the region to have a larger than normal storm water retention capacity for big wetting events, e.g., by root enhanced infiltration and organic matter facilitated soil hydrophilicity (32-34). Following the wetting episode, this increasing vegetation cover tends to demand more root water uptake, which gradually depletes soil moisture, and thus TWS (the upper blue arrow in Fig. 5). This process reverses the previous positive TWS trend to a negative one.

In contrast, for a region with a decreasing TWS (the upper blue arrow in Fig. 5) during a seesaw period, vegetation cover will reduce. Such a surface tends to favor runoff generation and soil erosion, leading to a lower storm water retention (35-37). Since Australia is prone to wildfires, the storm water retention can be further weakened by fire-induced hydrophobic soils (38). After the wetting episode, the previously reduced vegetation cover demands less soil moisture, leading to a gradual increase of TWS (the lower blue arrow in Fig. 5) during the interval period. 
The above two situations explain the seesaw resetting pattern in which a positive (negative) seesaw stage is reset to a negative (positive) one after a short wetting episode. In the last three interval periods precipitation shows opposite trends between the eastern and western parts of Australia consistent with TWS, vegetation recovering (degrading) might be facilitated by increasing (decreasing) precipitation. However, no significant difference of changes in regional average precipitation anomaly between eastern and western Australia is observed in any interval period (Fig. S2 (b)). It suggests that vegetation and soil moisture interactions are very likely the primary mechanism for the observed TWS seesaws.

By comparing the 12-month average NDVI anomaly immediately before (Fig. 6 (a-c)) and 12-month average TWS anomaly immediately after (Fig. $6(\mathrm{~d}-\mathrm{f})$ ) the three La Niña induced continent-wide wetting episodes shown in Fig. 2 (b), we can see that the half of the continent (either east or west) with increased vegetation cover (positive NDVI anomaly) prior to a La Niña triggered wetting episode, retains larger than normal storm water (positive TWS anomaly) after the episode. After a wetting episode, the part dominated by positive NDVI and TWS anomalies experiences a decreasing evapotranspiration (ET) (Fig. $6(\mathrm{~g}-\mathrm{h})$ ), reflecting a gradually decreasing vegetation cover resulting from a depleting TWS. These processes are consistent with the trends of NDVI and TWS during the corresponding interval period. In addition, vegetation in the region with positive (negative) $N D V I$ anomaly prior to the last La Niña induced continent-wide wetting shows decreasing (increasing) trend during Mar 2013-Dec 2018 (Fig. S3). Such results support the mechanism of the Australia TWS seesaw phenomenon we explained above.

Based on this understanding, we suppose that vegetation dynamic, in response to continent-wide wetting, plays an important role in the TWS seesaw in Australia. However, the interval period should be long enough for woody vegetation to recover in one part while to degrade in the other part of Australia, so that TWS seesaw between the two parts could be reset by a La Niña induced continent-wide wetting.

\section{Conclusion}

This study, based on three TWS datasets (14-year original JPL GRACE TWS and 31- and 114-year reconstructed GRACE TWS), elucidates a new spatio-temporal pattern of wetting-drying over Australia. Four continuous seesaw wetting and drying phases between eastern and western Australia are observed in the past five decades, which is characterized by eastern Australia gaining water while the western part is losing water, and vice versa. Strong La Niña induced continent-wide wetting, resets this pattern, leaving each seesaw to last for $11 \pm 5$ years. The TWS seesaw phenomenon is substantiated by a similar pattern in NDVI between eastern and western Australia during Feb 1990-Jul 1998 and Jul 2002-Jul 2010. This continental scale TWS seesaw pattern seems to be resulting from woody vegetation response to climate variability and its feedback on hydrological processes.

The results of this study contribute to a better understanding of drying and wetting phases and hence can stimulate an adaptive forest, water, and disaster risk management in the wake of a strong La Niña induced continent-wide wetting in Australia. Our finding suggests that at the end of a seesaw drying phase, poor vegetation cover limits the landscape water retention capacity. Hence, during a drying phase a reasonable management response might be to increase storm water harvesting capacity. At the beginning of a seesaw drying phase, previously increased vegetation cover (resulting from a previous seesaw wetting) depletes root zone moisture, leading to landscape degradation in the subsequent years. Reducing vegetation cover right after the wetting episode might reduce the risk of heatwaves and bushfires in the later dry stage. 


\section{Materials and Methods}

Datasets. Three TWS datasets are applied in this study. The first is the original GRACE TWS dataset (RL06M.MSCNv01) $(17,18)$ from 2003 to 2016 provided by the NASA Jet Propulsion Laboratory (JPL). Seventeen months of missing TWS data (June 2003, January 2011, June 2011, May 2012, October 2012, March 2013, August 2013, September 2013, February 2014, July 2014, December 2014, June 2015, October 2015, November 2015, April 2016, September 2016 and October 2016) are filled by linear interpolation using the months either side $(39,40)$. In order to investigate the wetting and drying cycles over a long period, two reconstructed GRACE TWS datasets for 1985-2015 (19) and for 1901-2014 (20) are also applied. Statistical data-driven models, calibrated with observations, are used to reconstruct the TWS dataset of 1901-2014, which perform well compared with state-of-the-art hydrological models $(20,41)$. The seasonality of TWS has been removed, which is represented by the use of a TWS anomaly in this study. GIMMS NDVI dataset (42, 43) is available from July 1981 to December 2015. Moderate Resolution Imaging Spectroradiometer (MODIS) $N D V I$ dataset (44) is available from March 2000. Both TWS and NDVI data are used at a $0.5^{\circ} \times 0.5^{\circ}$ spatial resolution. GLDAS_NOAH025_M evapotranspiration data $(45,46)$ are used at a $0.25^{\circ} \times 0.25^{\circ}$ spatial resolution. AWAP soil moisture and precipitation data $(29,30)$ are of $0.05^{\circ} \times 0.05^{\circ}$ spatial resolution.

Climate indices. Two ocean-atmosphere indices including Southern Oscillation Index (SOI) (47-49) and Indian Ocean Dipole Mode Index (DMI) (50) are applied in this study since they play an important role in Australia's hydroclimatic variations $(11,15,51)$.

EOFs. The empirical orthogonal functions (EOFs) decompose a space-time field into spatial patterns and associated temporal signals. For a continuous space-time field $\operatorname{TWS}(t, s), t$ and $s$ denote respectively time and spatial position, the decomposition is described as in the study of Hannachi et al. (52):

$$
\operatorname{TWS}(t, s)=\sum_{k=1}^{K} c_{k}(t) u_{k}(s)
$$

where $K$ is the number of modes included in the field, using an optimal set of basis functions of space $u_{k}(s)$ and expansion functions of time $c_{k}(t)$. For the space-time field of TWS over Australia, the EOFs method finds a set of orthogonal spatial patterns $\left(E O F_{k}\right)$ along with a set of associated uncorrelated time series or principal components $\left(P C_{k}\right)$ (i.e., $P C 1$ corresponds to EOF1 and so on). This method also provides the explained variance, which indicates how much variability of TWS is explained by each decomposed mode $\left(E O F_{k} / P C_{k}\right)$.

Significance test. The significance of linear trend is tested by the Mann-Kendall (M-K) test $(53,54)$. Analysis of covariance is applied to test the significance of difference between two time series. The 0.05 significance level is adopted in this study.

\section{Acknowledgments}

The datasets used in this study are available online in the following locations: JPL GRACE TWS, https://grace.jpl.nasa.gov/; reconstructed TWS from 1985-2015 (GRACE_REC_v01), http://rossa-prodap21.ethz.ch/delivery/DeliveryManagerServlet?dps_pid=IE5766472; reconstructed TWS from 1901-2014 (GRACE_REC_v03), https://doi.org/10.6084/m9.figshare.7670849; GIMMS NDVI, https://climatedataguide.ucar.edu/climate-data/ndvi-normalized-difference-vegetation-index-3rd-generationnasagfsc-gimms; MODIS NDVI, https://neo.sci.gsfc.nasa.gov/view.php?datasetld=MOD_NDVI_M; GLDAS_NOAH ET, 
https://disc.gsfc.nasa.gov/datasets/GLDAS_NOAH025_M_2.0/summary?keywords=GLDAS; SOI index, https://www.esrl.noaa.gov/psd/gcos_wgsp/Timeseries/SOI/; DMI index, https://www.esrl.noaa.gov/psd/gcos_wgsp/Timeseries/DMI/. AWAP soil moisture and precipitation data are viewable at: http://www.csiro.au/awap and downloadable via ftp by application to Peter.Briggs@csiro.au. We are thankful to their provision of data. The first author's PhD scholarship has been provided by the China Scholarship Council, Ministry of Education, China, and Flinders University, Australia.

\section{References}

1. N. Nicholls, W. Drosdowsky, B. Lavery, Australian rainfall variability and change. Weather. 52, 66-72 (1997).

2. R. Dey, S. C. Lewis, J. M. Arblaster, N. J. Abram, A review of past and projected changes in Australia's rainfall. WIREs. Clim. Change. 10, 1-23 (2019).

3. A. Ahlström et al., The dominant role of semi-arid ecosystems in the trend and variability of the land $\mathrm{CO}_{2}$ sink. Science. 348, 895-899 (2015).

4. Z. Xie et al., Spatial partitioning and temporal evolution of Australia's total water storage under extreme hydroclimatic impacts. Remote Sens. Environ. 183, 43-52 (2016).

5. F. Johnson et al., Natural hazards in Australia: floods. Clim. Change. 139, 21-35 (2016).

6. A. S. Kiem et al., Natural hazards in Australia: droughts. Clim. Change. 139, $37-54$ (2016).

7. X. Ma, A. Huete, S. Moran, G. Ponce-Campos, D. Eamus, Abrupt shifts in phenology and vegetation productivity under climate extremes. J. Geophys. Res. Biogeosci. 120, 2036-2052 (2015).

8. M. Zhao, S. W. Running, Drought-induced reduction in global terrestrial net primary production from 2000 through 2009. Science. 329, 940-943(2010).

9. B. Poulter et al., Contribution of semi-arid ecosystems to interannual variability of the global carbon cycle. Nature. 509, 600-603 (2014).

10. M. Heberger, "Australia's Millennium Drought: Impacts and Responses" in The World's Water. Island Press, Washington, DC. P. H. Gleick Eds. (Springer, 2012), pp. 97-125.

11. A. Van Dijk et al., The Millennium Drought in southeast Australia (2001-2009): Natural and human causes and implications for water resources, ecosystems, economy, and society. Water. Resour. Res. 49, 1040-1057 (2013).

12. J. J. Sharples et al., Natural hazards in Australia: extreme bushfire. Clim. Change. 139, 85-99 (2016).

13. S. E. Perkins-Kirkpatrick et al., Natural hazards in Australia: heatwaves. Clim. Change. 139, 101 (2016).

14. N. Herold, J. Kala, L. V. Alexander, The influence of soil moisture deficits on Australian heatwaves. Environ. Res. Lett. 11, 064003 (2016).

15. A. S. Kiem, D. C. Verdon-Kidd, Towards understanding hydroclimatic change in Victoria, Australiapreliminary insights into the "big dry". Hydrol. Earth. Syst. Sci. 14, 433-445 (2010).

16. N. Nicholls, What caused the eastern Australia heavy rains and floods of 2010/11. Aust. Meteorol. Ocean. 24, 33-34 (2011).

17. M. M. Watkins, D. N. Wiese, D. Yuan, C. Boening, F. W. Landerer, Improved methods for observing Earth's time variable mass distribution with GRACE using spherical cap mascons. J. Geophys. Res. Solid Earth. 120, 2648-2671, (2015).

18. D. N. Wiese, F. W. Landerer, M. M. Watkins, Quantifying and reducing leakage errors in the JPL RL05M GRACE mascon solution. Water Resour. Res. 52, 7490-7502, (2016). 
19. V. Humphrey, L. Gudmundsson, S. I. Seneviratne, A global reconstruction of climate-driven subdecadal water storage variability. Geophys. Res. Lett. 44, 2300-2309 (2017).

20. V. Humphrey, L. Gudmundsson, GRACE-REC: a reconstruction of climate-driven water storage changes over the last century. Earth Syst. Sci. Data. 11(3), 1153-1170 (2019).

21. M. J. Salinger, J. A. Renwick, A. B. Mullan, Interdecadal Pacific Oscillation and South Pacific climate. Int. J. Climatol. 21, 1705-1721 (2001).

22. A. Gershunov, T. P. Barnett, Interdecadal modulation of ENSO teleconnections. Bull. Am. Meteorol. Soc. 79, 2715-2725 (1998).

23. S. Power, T. Casey, C. Folland, A. Colman, V. Mehta, Inter-decadal modulation of the impact of ENSO on Australia. Clim. Dyn. 15, 319-324 (1999).

24. H. Guan, E. R. Vivoni, J. L. Wilson, Effects of atmospheric teleconnections on seasonal precipitation in mountainous regions of the southwestern U.S.: A case study in northern New Mexico. Geophys. Res. Lett. 32, L23701, (2005).

25. W. Cai, P. Van Rensch, The 2011 southeast Queensland extreme summer rainfall: A confirmation of a negative Pacific Decadal Oscillation phase? Geophys. Res. Lett. 39, L08702 (2012).

26. A. D. King, L. V. Alexander, M. G. Donat, Asymmetry in the response of eastern Australia extreme rainfall to low-frequency Pacific variability. Geophys. Res. Lett. 40 (10), 2271-2277 (2013).

27. J. S. Risbey, M. J. Pook, P. C. McIntosh, M. C. Wheeler, H. H. Hendon, On the remote drivers of rainfall variability in Australia. Mon. Wea. Rev. 137, 3233-3253 (2009).

28. W. Cai, A. Purich, T. Cowan, P. Van Rensch, E. Weller, Did climate change-induced rainfall trends contribute to the Australian millennium drought? J. Climate. 27, 3145-3168 (2014).

29. M. R. Raupach et al., Australian Water Availability Project (AWAP): CSIRO Marine and Atmospheric Research Component: Final Report for Phase 3. CAWCR Technical Report No. 013. (CAWCR, 2009).

30. M. R. Raupach et al., Australian Water Availability Project, Data Release 26m, CSIRO Oceans and Atmosphere, Canberra, Australia. (2018).

31. M. Rodell, J. S. Famiglietti, An analysis of terrestrial water storage variations in Illinois with implications for the Gravity Recovery and Climate Experiment (GRACE). Water. Resour. Res. 37(5), 1327-1339 (2001).

32. B. Lange, P. Luescher, P. F. Germann, Significance of tree roots for preferential infiltration in stagnic soils. Hydrol. Earth Syst. Sci. 13, 1809-1821 (2009).

33. H. Guan, J. Simunek, B. D. Newman, J. L. Wilson, Modelling investigation of water partitioning at a semiarid ponderosa pine hillslope. Hydrol. Process. 24, 1095-1105 (2010).

34. C. Wang, C. Zhao, Z. Xu, Y. Wang, H. Peng, Effect of vegetation on soil water retention and storage in a semi-arid alpine forest catchment. J. Arid. Land. 5, 207-219 (2013).

35. G. Dunjó, G. Pardini, M. Gispert, The role of land use-land cover on runoff generation and sediment yield at a microplot scale, in a small Mediterranean catchment. J. Arid Environ. 57(2), 239-256 (2004).

36. B. P. Kothyari, P. K. Verma, B. K. Joshi, U.C. Kothyari, Rainfall-runoff-soil and nutrient loss relationships for plot size areas of bhetagad watershed in Central Himalaya, India. J. Hydrol. 293 (14), 137-150 (2004).

37. A. G. Mohammad, M. A. Adam, The impact of vegetative cover type on runoff and soil erosion under different land uses. Catena. 81(2), 97-103 (2010).

38. J. Mataix-Solera, A. Cerdà, V. Arcenegui, A. Jordán, L. M. Zavala, Fire effects on soil aggregation: A review. Earth-Sci. Rev. 109 (1-2), 44-60 (2011). 
39. D. Long et al., Deriving scaling factors using a global hydrological model to restore GRACE signals. Remote. Sens. Environ. 168, 177-193 (2015).

40. R. Andrew, H. Guan, O. Batelaan, Estimation of GRACE water storage components by temporal decomposition. J. Hydrol. 552, 341-350 (2017).

41. R. S. Padrón et al., Observed changes in dry-season water availability attributed to human-induced climate change. Nat. Geosci. 13, 477-481 (2020).

42. C. J. Tucker et al., An extended AVHRR-8km NDVI dataset compatible with MODIS and SPOT vegetation NDVI data. Int. J. Remote Sens. 26, 4485-4498 (2005).

43. J. E. Pinzon, C. J. Tucker, A Non-Stationary 1981-2012 AVHRR NDVI3g Time Series. Remote. Sens. 6, 6929-6960 (2014).

44. K. Didan, A. Barreto Munoz, R. Solano, A. Huete, MODIS Vegetation Index Users Guide. (2017).

45. M. Rodell et al., The Global Land Data Assimilation System. Bull. Amer. Meteor. Soc. 85, 381-394 (2004).

46. H. Beaudoing, M. Rodell, NASA/GSFC/HSL (2015), GLDAS Noah Land Surface Model L4 monthly $0.25 \times 0.25$ degree V2.0, Greenbelt, Maryland, USA, Goddard Earth Sciences Data and Information Services Center (GES DISC), Accessed: [Data Access Date], 10.5067/9SQ1B3ZXP2C5.

47. C. F. Ropelewski, P. D. Jones, An extension of the Tahiti-Darwin Southern Oscillation Index. Mon. Weather. Rev. 115, 2161-2165 (1987).

48. R. J. Allan, N. Nicholls, P. D. Jones, I. J. Butterworth, A further extension of the Tahiti-Darwin SOI, early SOI results and Darwin pressure. J. Climate. 4, 743-749 (1991).

49. G. P. Können, P. D. Jones, M. H. Kaltofen, R. J. Allan, Pre-1866 extensions of the Southern Oscillation Index using early Indonesian and Tahitian meteorological readings. J. Climate. 11, 23252339 (1998).

50. N. H. Saji, T. Yamagata, Possible impacts of Indian Ocean Dipole mode events on global climate. Clim. Res. 25 (2), 151-169 (2003).

51. D. C. Verdon-Kidd, A. S. Kiem, On the relationship between large-scale climate modes and regional synoptic patterns that drive Victorian rainfall. Hydrol. Earth Syst. Sci. 13(4), 467-479 (2009).

52. A. Hannachi, I. T. Jolliffe, D. B. Stephenson, Empirical orthogonal functions and related techniques in atmospheric science: a review. Int. J. Climatol. 27(9), 1119-1152 (2007).

53. H. B. Mann, Nonparametric tests against trend. Econometrica. 13 (3), 245-259 (1945).

54. M. G. Kendall, Rank Correlation Methods. Oxford: Griffin. (1975). 
Figures and Tables

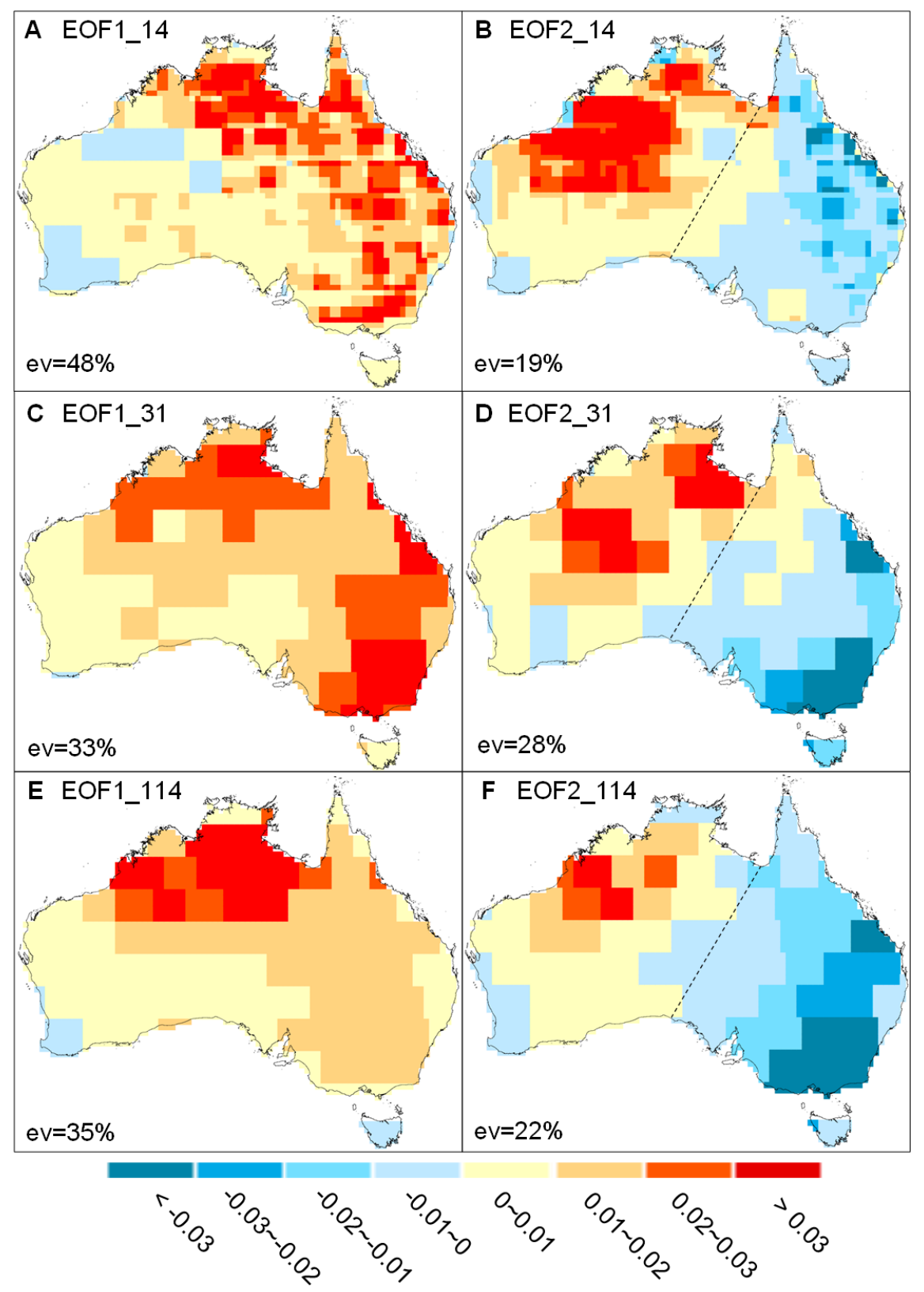

Figure 1. Spatial patterns of the first two modes (EOF1 \& EOF2) of Australia TWS variation. (A-B) 14-year original JPL GRACE TWS (2003-2016), (C-D) 31-year reconstructed GRACE TWS (1985-2015), (E-F) 114 year reconstructed GRACE TWS (1901-2014). Acronym "ev" stands for explained variance. An east-west opposite (EOF2) pattern is observed in all three datasets. 

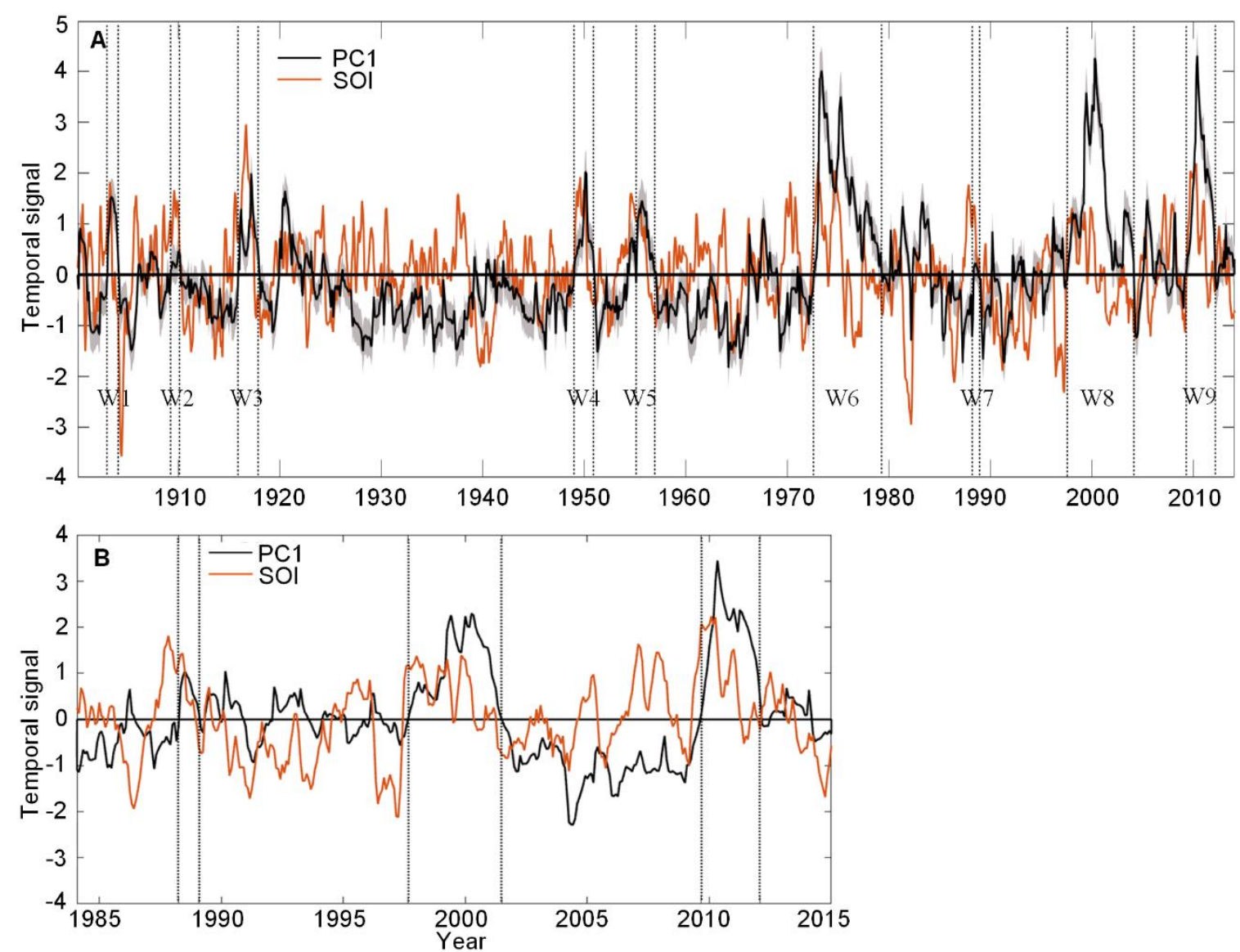

Figure 2. La Niña induced continent-wide wetting episodes identified based on $\mathrm{SO}$ and the temporal signal of the first mode (PC1) of Australia TWS variation. (A) PC1 vs. SOI during 1901-2014 ( $r=0.18, p<0.05)$. The black curve indicates mean $P C 1$ derived from $100 P C 1$ members and the shaded areas represent \pm 1 standard deviation of $100 P C 1$ time series derived from the ensemble members. A 3-month moving average was applied to $S O I$ for readability (orange curve). The periods marked by a pair of dashed lines denote the $L a$ Niña (positive SOI) induced continent-wide wetting episodes (positive PC1) (W1: Dec 1903-Jan 1905, W2: Mar 1910-Feb 1911, W3: Nov 1916-Nov 1918, W4: Dec 1949-Nov 1951, W5: Feb 1956-Dec 1957, W6: Jul 1973-Mar 1980, W7: Apr 1989-Sep 1989, W8: Aug 1998-Jun 2002, W9: Apr 2010- Jan 2013), which are identified according to the time points when average TWS anomaly in Australia starts to increase, triggered by strong La Niña, and when it returns to equilibrium. (B) PC1 vs. SOI during 1985-2015 ( $r=0.23, p<0.05)$. The three marked La Niña induced continent-wide wetting episodes (Apr 1989-Jan 1990, Aug 1998-Jun 2002, and Aug 2010- Feb 2013) respectively correspond to W7, W8 and W9 shown in (A). 

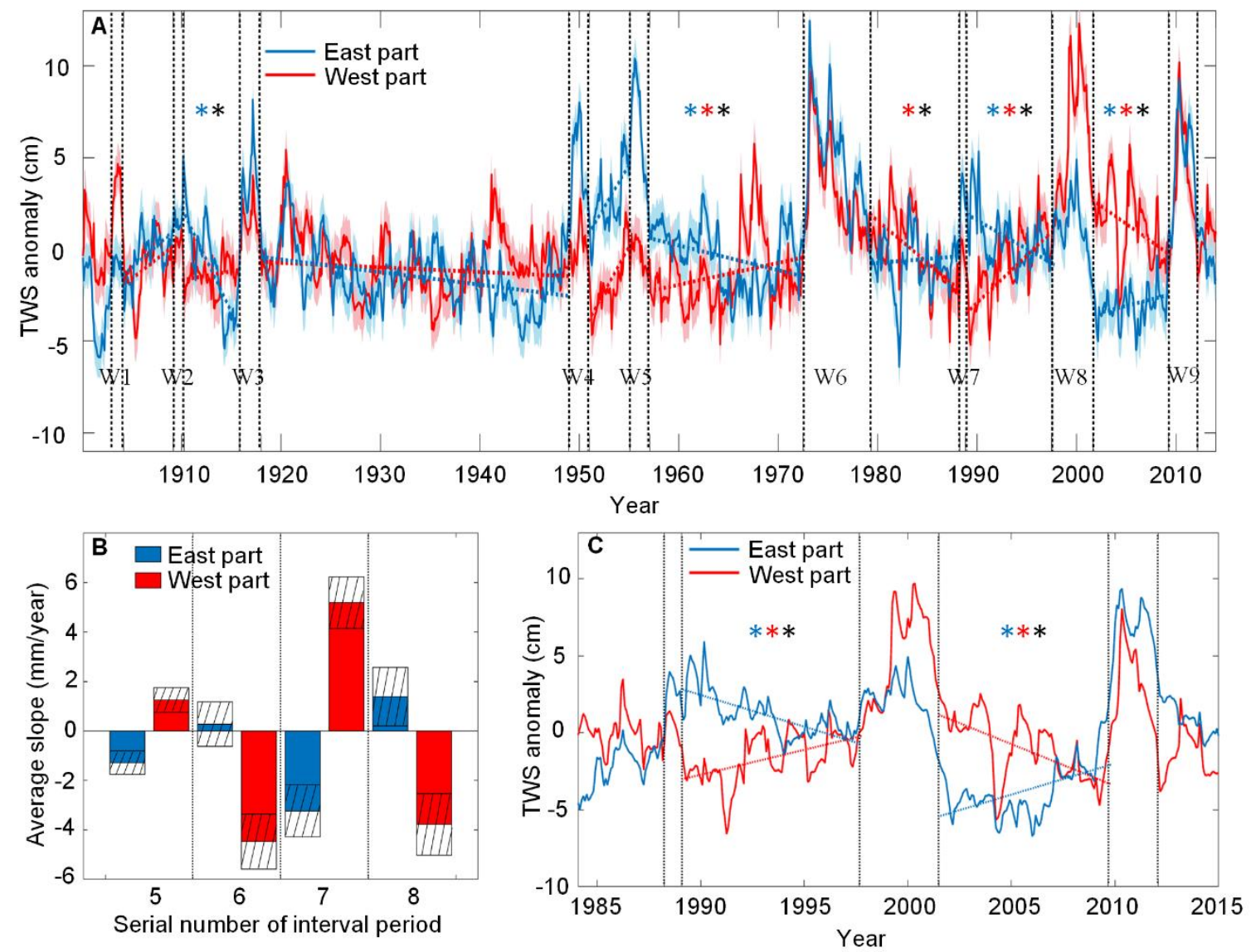

Figure 3. TWS seesaw between eastern and western Australia. (A) Regional average TWS anomaly of eastern and western Australia during 1901-2014 (the boundary of eastern and western parts is shown in Fig. 1). Uncertainty (shaded area) is quantified by \pm 1 standard deviation derived from 100 ensemble members. Opposite linear trends of regional average TWS anomaly of eastern and western Australia are observed for Mar 1911-Oct 1916, Jan 1958-Jun 1973, Apr 1980-Mar 1989, Oct 1989-Jul 1998 and Jul 2002-Mar 2010 (blue (red) asterisk $\left({ }^{*}\right)$ indicates the trend of eastern (western) Australia is significant at 0.05 significance level, and the black asterisk indicates the changes of regional average TWS anomaly between eastern and western Australia are significantly different); (B) slopes of regional average TWS anomaly derived from 100 ensemble members in the last four interval periods (5 8). Hatched bars indicate uncertainty ( \pm 1 standard deviation) in trends. Within all the four interval periods, average trends of the East and West part are significantly different at 0.05 significance level. (C) same as (A) but based on TWS data of 1985-2015. Opposite linear trends of regional average TWS anomaly of the East and West part are observed during Feb 1990-Jul 1998 and Jul 2002- Jul 2010. 


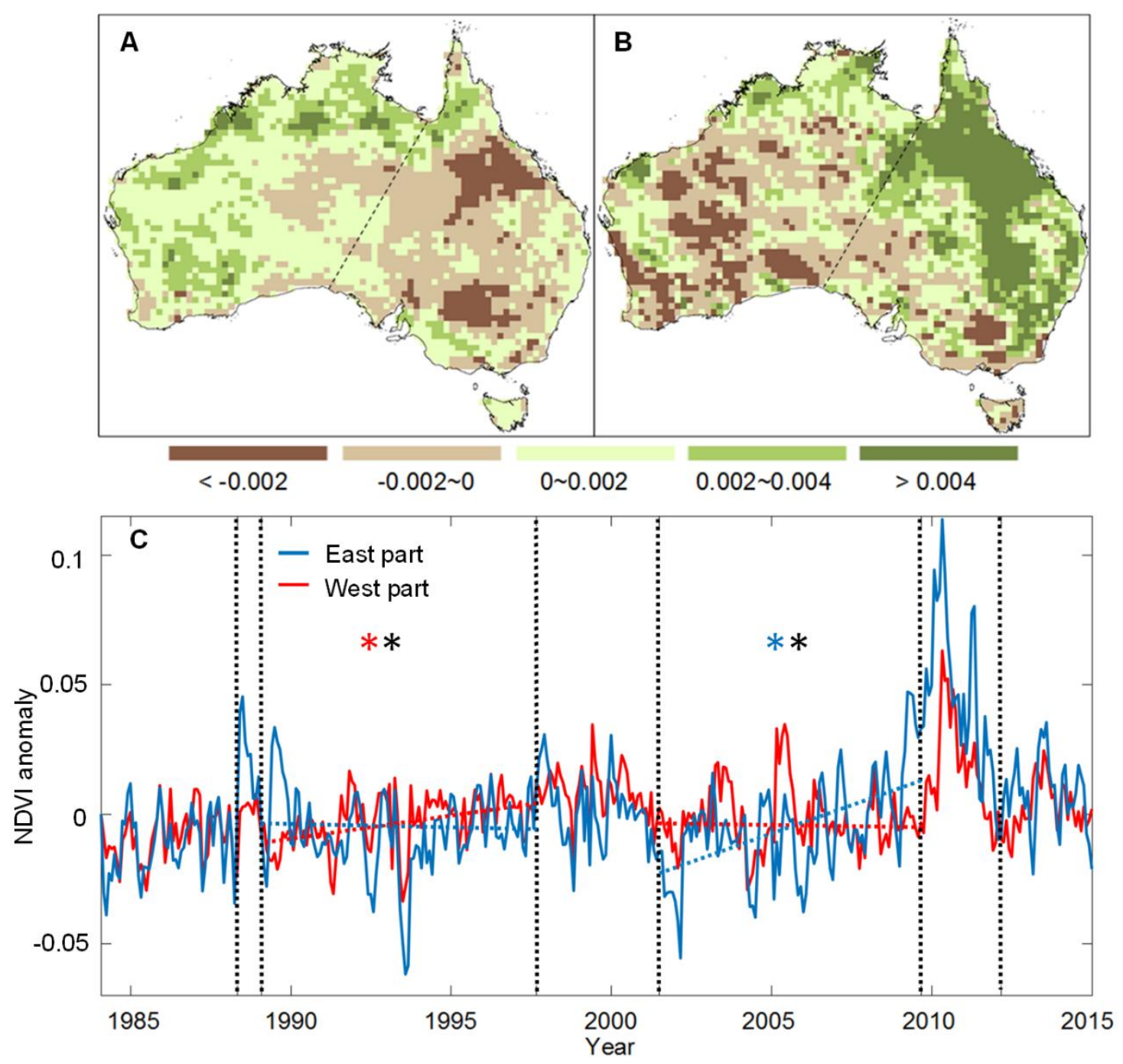

Figure 4. Seesaw phenomenon of NDVI during 1985-2015. (A-B) Linear trends (per month) of NDVI during two interval periods (Feb 1990-Jul 1998 and Jul 2002- Jul 2010); (C) same as Fig. 3 (C) but for NDVI anomaly. Opposite linear trends of regional average $N D V I$ anomaly of eastern and western Australia are observed during Feb 1990-Jul 1998 and Jul 2002- Jul 2010. Two upward trends are significant at 0.05 significance level while two downward trends are non-significant. Changes of regional average NDVI anomaly between the two parts are significantly different in both interval periods. 


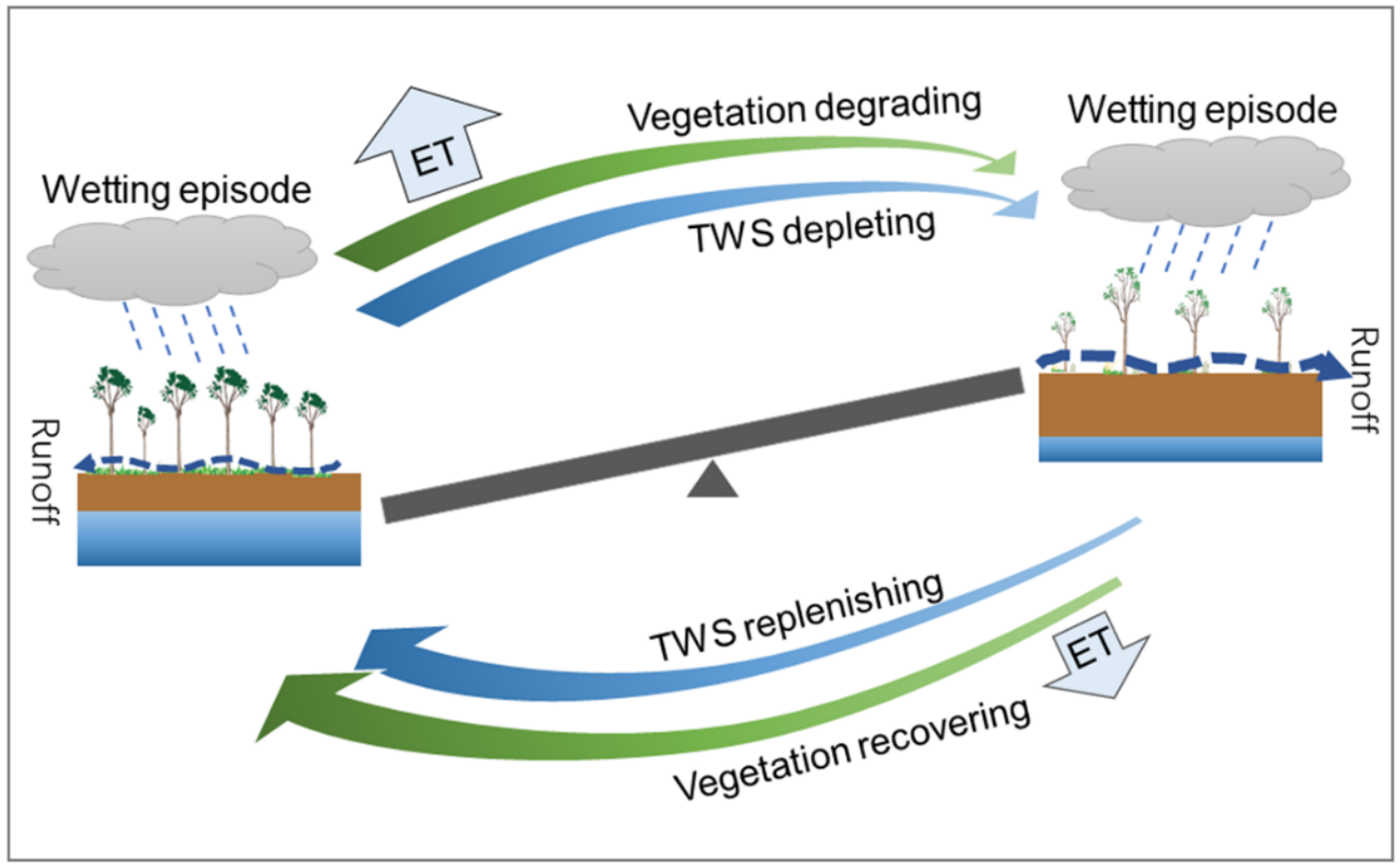

Figure 5. A schematic showing possible terrestrial water storage and vegetation interactions, leading to TWS seesaw and its resetting by big wetting episodes. 

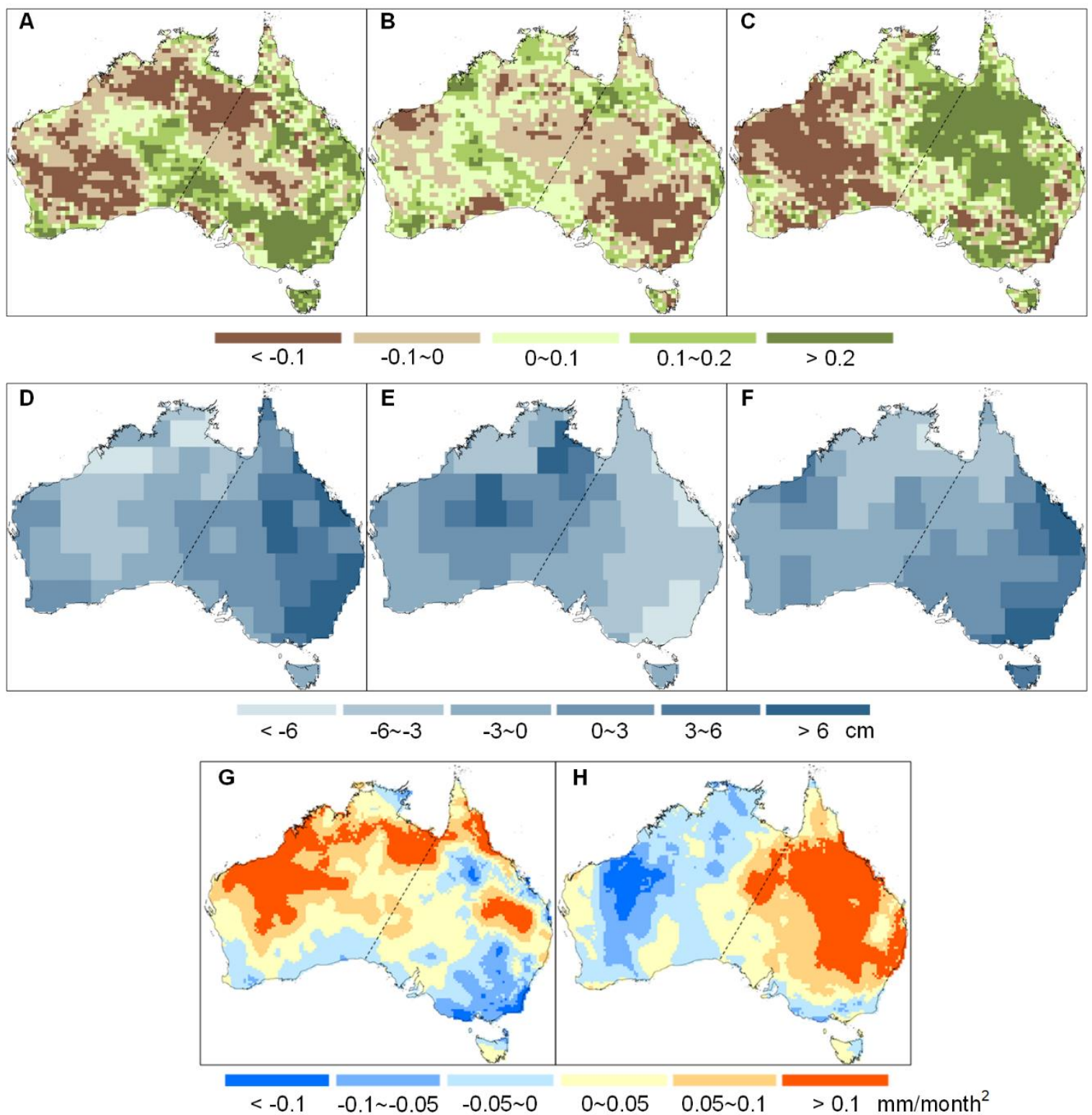

Figure 6. Evidence for the possible mechanism of the TWS seesaw phenomenon. Average 12-month NDVI anomaly (A-C) immediately before and average 12-month TWS anomaly (D-F) immediately after the three continent-wide wetting episodes: Apr 1989-Jan 1990, Aug 1998-Jun 2002, and Aug 2010- Feb 2013; (G-H) linear trends of evapotranspiration (ET) during two interval periods: Feb 1990-Jul 1998 and Jul 2002- Jul 2010. 


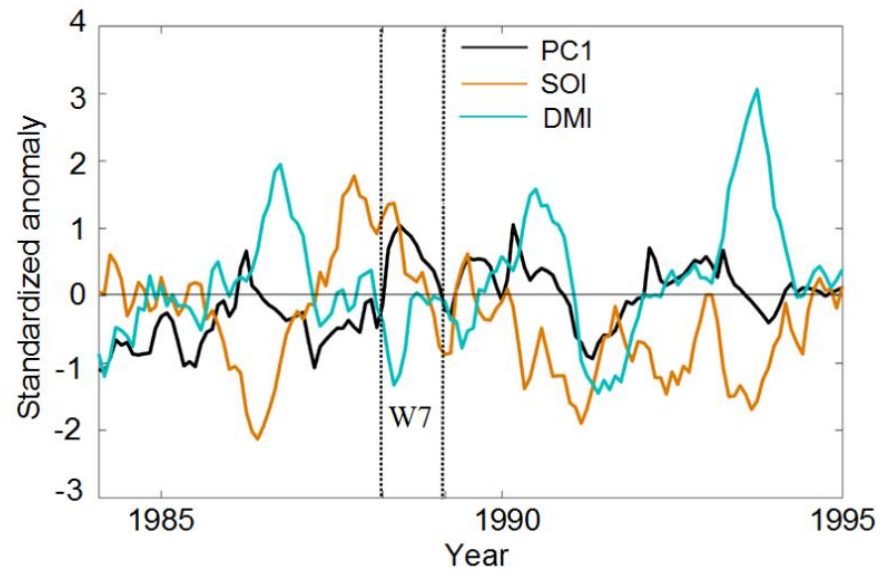

Fig. S1. Plot of $P C 1$, monthly SOI and DMI from 1985 to 1995. 

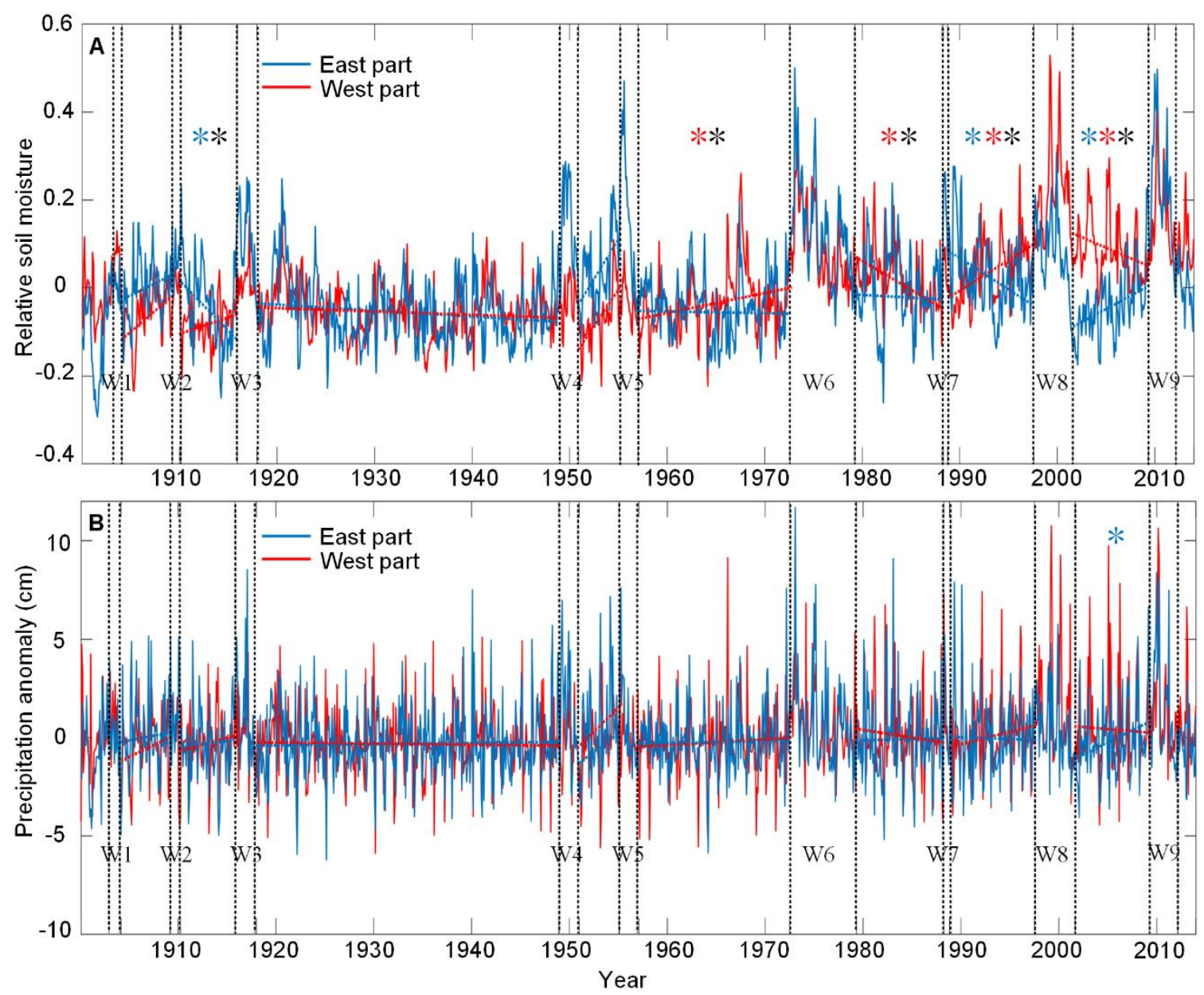

Fig. S2. (A) Regional average soil moisture of eastern and western Australia during 1901-2014, which shows a similar seesaw pattern to TWS (changes of regional average soil moisture between eastern and western Australia are significantly different in five interval periods: Mar 1911-Oct 1916, Jan 1958-Jun 1973, Apr 1980Mar 1989, Oct 1989-Jul 1998 and Aug 2002-Mar 2010; (B) same as (A) but for precipitation, opposite trends are observed in the last three interval periods, but no significant difference of changes in regional average precipitation anomaly between eastern and western Australia is observed in any interval period. 

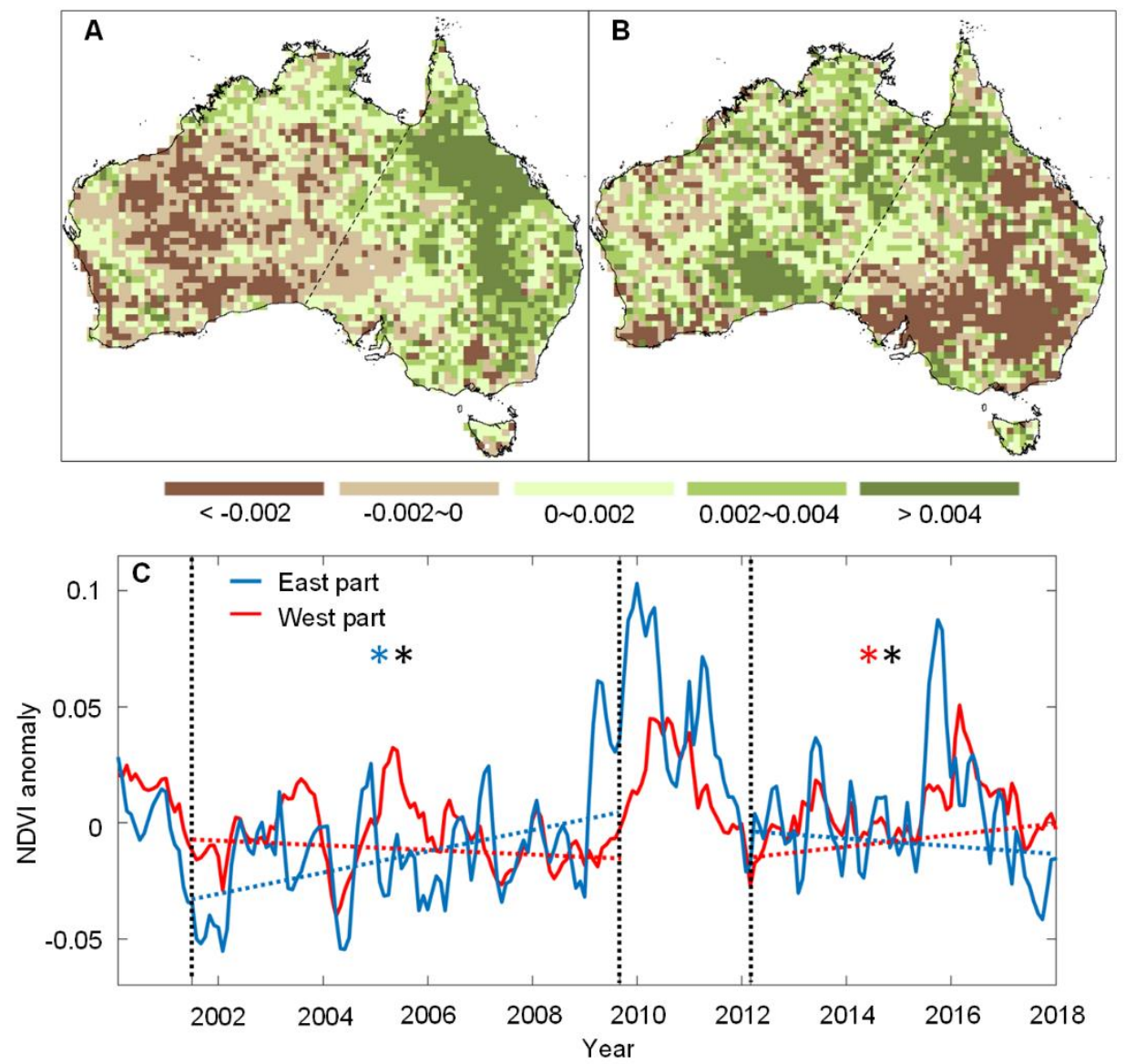

Fig. S3. (A-B) Linear trends (per month) of MODIS NDVI during Jul 2002-Jul 2010 and Mar 2013-Dec 2018; (C) regional average NDVI anomaly of eastern and western Australia during 2001-2018. Results from GIMMS (Fig. 4) and MODIS NDVI datasets are consistent during the overlapped interval Jul 2002-Jul 2010. Opposite linear trends between the two parts are also observed during Mar 2013-Dec 2018. Changes of regional average MODIS NDVI anomaly between eastern and western Australia are significantly different in both interval periods. 